\title{
Abkürzungen der Übersetzersprachen
}

\begin{tabular}{|c|c|c|c|}
\hline afr & Afrikaans & kelt & Keltisch \\
\hline agr & Altgriechisch & kopt & Koptisch \\
\hline agr (Koine) & Altgriechisch (Koine) & korean & Koreanisch \\
\hline ahd & Althochdeutsch & kroat & Kroatisch \\
\hline alb & Albanisch & kurd & Kurdisch \\
\hline altägypt & Altägyptisch & ladin & Ladinisch \\
\hline altarab & Altarabisch & lat & Latein \\
\hline altisl & Altisländisch & lateinam & Lateinamerikanisch \\
\hline altndt & Altniederdeutsch & lett & Lettisch \\
\hline altper & Altpersisch & litau & Litauisch \\
\hline altslaw & Altslawisch & $\operatorname{lux}$ & Luxemburgisch \\
\hline am & Amerikanisches Englisch & mak & Makedonisch \\
\hline anord & Altnordisch & mhd & Mittelhochdeutsch \\
\hline arab & Arabisch & mongol & Mongolisch \\
\hline arm & Armenisch & ndl & Niederländisch \\
\hline bask & Baskisch & ndt & Niederdeutsch \\
\hline berndt & Berndeutsch & ndt (ostfäl.) & Niederdeutsch (Ostfälisch) \\
\hline bosn & Bosnisch & neugr & Neugriechisch \\
\hline breton & Bretonisch & ngr & Neugriechisch \\
\hline bulg & Bulgarisch & nor & Norwegisch \\
\hline chin & Chinesisch & nordfries & Nordfriesisch \\
\hline chin (klass) & Chinesisch (Klassisches) & obersorb-wend & Obersorbisch-Wendisch \\
\hline dän & Dänisch & ostfries & Ostfriesisch \\
\hline demot & Demotisch & pers & Persisch \\
\hline engl & Englisch & plattdt & Plattdeutsch \\
\hline engl (afrik) & Englisch (Afrikanisches) & poln & Polnisch \\
\hline engl (austral) & Englisch (Australisches) & port & Portugiesisch \\
\hline engl (ind) & Englisch (Indisches) & port (afrik) & Portugiesisch (Afrikanisches) \\
\hline engl (ir) & Englisch (Irisches) & port (bras) & Portugiesisch (Brasilianisches) \\
\hline engl (kanad) & Englisch (Kanadisches) & prov & Provenzalisch \\
\hline engl (karib) & Englisch (Karibisches) & rät & Rätoromanisch \\
\hline engl (scots) & Englisch (Schottisches) & rum & Rumänisch \\
\hline engl (walis) & Englisch (Walisisches) & russ & Russisch \\
\hline esp & Esperanto & sanskr & Sanskrit \\
\hline estn & Estnisch & schott (gälisch) & Schottisch-Gälisch \\
\hline färö & Färöisch & schott (Lowlands) & Lowlandschottisch \\
\hline finn & Finnisch & schw & Schwedisch \\
\hline fläm & Flämisch & serb & Serbisch \\
\hline friaul & Friaulisch & serbokroat & Serbokroatisch \\
\hline fries & Friesisch & siebenbürg.- & Siebenbürgisches Sächsisch \\
\hline frz & Französisch & sächs & \\
\hline frz (kanad) & Französisch (Kanadisches) & skand & Skandinavische Sprachen \\
\hline frz (nordafrik) & Französisch (Nordafrikanisches) & slowak & Slowakisch \\
\hline galic & Galicisch & slowen & Slowenisch \\
\hline georg & Georgisch & sorb & Sorbisch \\
\hline $\mathrm{gr}$ & Griechisch & span & Spanisch \\
\hline hebr & Hebräisch & $\operatorname{span}(\arg )$ & Spanisch (Argentinisches) \\
\hline holl & Holländisch & $\operatorname{span}(\operatorname{mex})$ & Spanisch (Mexikanisches) \\
\hline indon & Indonesisch & span (venez) & Spanisch (Venezolanisches) \\
\hline ir & Irisch & tsch & Tschechisch \\
\hline isl & Isländisch & türk & Türkisch \\
\hline ital & Italienisch & tuwin & Tuwinisch \\
\hline jap & Japanisch & $\mathrm{ukr}$ & Ukrainisch \\
\hline jidd & Jiddisch & ung & Ungarisch \\
\hline kat & Katalanisch & usbek & Usbekisch \\
\hline
\end{tabular}




\section{Abkürzungen}

vietn

wareng weißruss
Vietnamesisch

Warengisch

Weißrussisch wogul

zürichdt

Wogulisch

Zürichdeutsch 